\title{
Chandra view of Abell 407: the central compact group of galaxies and the interaction between the radio AGN and the ICM
}

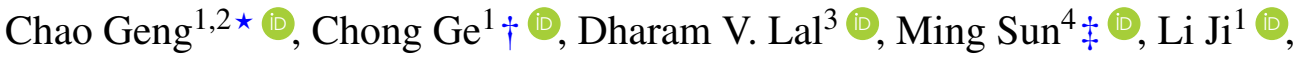

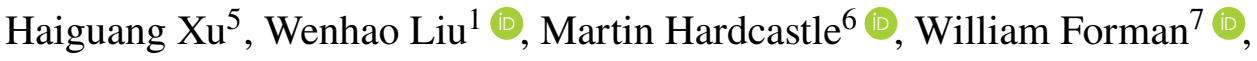 \\ Ralph $\mathrm{Kraft}^{7}{ }^{10}$, Christine Jones ${ }^{6}$ \\ ${ }^{1}$ Purple Mountain Observatory, Chinese Academy of Science, Nanjing 210034, People's Republic of China \\ ${ }^{2}$ Department of Astronomy, University of Science and Technology of China, Hefei 230026, People's Republic of China \\ ${ }^{3}$ National Centre for Radio Astrophysics, Tata Institute of Fundamental Research, Post Box 3, Ganeshkhind P.O., Pune 411007, India \\ ${ }^{4}$ Department of Physics and Astronomy, University of Alabama in Huntsville, Huntsville, AL 35899, USA \\ ${ }^{5}$ School of Physics and Astronomy, Shanghai Jiao Tong University, 800 Dongchuan Road, Minhang, Shanghai 200240, People's Republic of China \\ ${ }^{6}$ Centre for Astrophysics Research, School of Physics, Astronomy \& Mathematics, University of Hertfordshire, College Lane, Hatfield AL10 9AB, UK \\ ${ }^{7}$ Harvard-Smithsonian Center for Astrophysics, 60 Garden Street, Cambridge, MA 02138, USA
}

Accepted. Received; in original form

\begin{abstract}
Abell 407 (A407) is a unique galaxy cluster hosting a central compact group of nine galaxies (named as 'Zwicky's Nonet'; G1 - G9 in this work) within a $30 \mathrm{kpc}$ radius region. The cluster core also hosts a luminous radio active galactic nucleus (AGN), 4C 35.06 with helically twisted jets extending over $200 \mathrm{kpc}$. With a $44 \mathrm{ks}$ Chandra observation of A407, we characterize the $\mathrm{X}$-ray properties of its intracluster medium (ICM) and central galaxies. The mean X-ray temperature of A407 is $2.7 \mathrm{keV}$ and the $M_{200}$ is $1.9 \times 10^{14} M_{\odot}$. We suggest that A407 has a weak cool core at $r<60 \mathrm{kpc}$ scales and at its very center, $<1-2 \mathrm{kpc}$ radius, a small galaxy corona associated with the strong radio AGN. We also conclude that the AGN 4C 35.06 host galaxy is most likely G3. We suggest that the central group of galaxies is undergoing a 'slow merge' procedure. The range of the merging time-scale is $0.3 \sim 2.3 \mathrm{Gyr}$ and the stellar mass of the future brightest cluster galaxy (BCG) will be $7.4 \times 10^{11} M_{\odot}$. We find that the regions which overlap with the radio jets have higher temperature and metallicity. This is consistent with AGN feedback activity. The central entropy is higher than that for other clusters, which may be due to the AGN feedback and/or merging activity. With all these facts, we suggest that A407 is a unique and rare system in the local universe that could help us to understand the formation of a massive BCG.
\end{abstract}

Key words: galaxies: clusters: individual: Abell 407 - galaxies: clusters: intracluster medium - X-rays: galaxies: clusters galaxies: ISM - galaxies: elliptical and lenticular, cD

\section{INTRODUCTION}

Galaxy clusters are the most massive virialized systems in the Universe. They form hierarchically through the merger of smaller substructures (Voit 2005). Clusters not only provide cosmological constraints but also provide a test of structure formation scenarios (Kravtsov \& Borgani 2012). The cores of massive clusters are one of the densest galaxy environments in the Universe, and thus are expected to host the strongest dynamical evolution processes. The brightest cluster galaxy (BCG) is usually located at the minimum potential well of the cluster and lies close to the peak of the X-ray emission (Beers \& Geller 1983; Jones \& Forman 1984). They are the largest and most massive galaxies in the Universe and usually an order of magnitude brighter than typical elliptical galaxies (Von

\footnotetext{
$\star$ gengchao@pmo.ac.cn

$\dagger$ astrochongge@gmail.com

† ming.sun@uah.edu
}

Der Linden et al. 2007). They may also host the most massive supermassive black holes (SMBHs) in the Universe (McConnell et al. 2011; Thomas et al. 2016). Hence, they have a distinctive formation history (Dressler 1978).

There are several different formation scenarios for BCGs: (1) the continuous merging of galaxies in the cluster potential, known as 'galactic cannibalism' (Ostriker \& Tremaine 1975; Hausman \& Ostriker 1978); (2) direct cooling from the hot-gas halo onto a preexisting central galaxy (Cowie \& Binney 1977; Fabian 1994); (3) tidal stripping of cluster galaxies that pass near the cluster center (Tovmassian \& Andernach 2012); (4) early merging during cluster collapse (Merritt 1985; Tremaine 1990; West 1994; Dubinski 1998). However, previous works have shown that mergers of smaller non-star-forming early-type galaxies (so-called 'dry mergers') are the primary mechanism through which BCGs grow from $z \sim 1$ to the present day (van Dokkum 2005; Bell et al. 2006; De Lucia \& Blaizot 2007). Liu et al. (2009) find that the merging rate increases with both the galaxy mass and age, which leads to the large fraction of dry mergers in 
the nearby universe. Khochfar \& Silk (2009) suggest there are about $10 \% \sim 20 \%$ of massive galaxies with a dry merger in the last Gyr.

Abell 407 (A407; $z=0.0462$ ) contains a compact group of elliptical galaxies in a luminous matrix (Fig. 1). Several bright galaxies appear to be embedded in a diffuse optical halo within a region of diameter $\sim 60 \mathrm{kpc}$ (Schneider \& Gunn 1982). It possibly provides a unique laboratory for the ongoing formation of a giant $\mathrm{CD}$ galaxy. In addition, the BCG-hosted active galactic nucleus (AGN) has a strong influence via 'AGN feedback' on its surroundings. In the radio bands, A407 shows 400-kpc scale helically twisted jets (Shulevski et al. 2015; Biju et al. 2017). Thus, A407 also provides a great opportunity to study the interaction between the galaxy nuclear activity and the cluster environment.

In this article, we make a detailed study of this remarkable system. This paper is organized as follows. In Section 2, we summarise the previous optical and radio observations of A407. Section 3 presents the Chandra data reduction methods. Section 4 is the X-ray observation results. Section 5 is the discussion of the results. Section 6 is our summary. Throughout this article, we assume a cosmology with $H_{0}=70 \mathrm{~km} \mathrm{~s}^{-1} \mathrm{Mpc}^{-1}, \Omega_{\mathrm{m}}=0.3$, and $\Omega_{\Lambda}=0.7$. The redshift of A407 $z=0.0462$ gives $1^{\prime \prime}=0.907 \mathrm{kpc}$. Uncertainties quoted in this paper are $1 \sigma$.

\section{PREVIOUS OBSERVATIONS}

\subsection{Optical detection}

Fig. 1(b) shows the optical image of the central region of A407 from the Sloan Digital Sky Survey (SDSS). It shows a complex ensemble of at least nine galaxies within $\sim 1$ arcmin. The central galaxies are embedded in a diffuse and low surface brightness stellar halo. Table 1 lists the optical $K_{S}$ band magnitudes, line of sight velocities, and masses of these nine galaxies.

The compact group of galaxies in A407 was first noticed by Zwicky \& Zwicky (1971). Biju et al. (2017) proposed to name this extraordinary system as 'Zwicky's Nonet'. It was later studied in more detail by Schneider \& Gunn (1982) who suggest this puzzling system is a snapshot of a forming cD galaxy. They consider that it is unlikely to be a pre-existing central $\mathrm{cD}$ capturing multiple galaxies. Instead, the $\mathrm{cD}$ is being created as a result of tidal disruption/merger. In summary, a recent merger generated the large-scale envelope and dynamical friction caused the remaining galaxies to merge.

\subsection{Radio detection}

The compact group also hosts a moderately luminous radio source, 4C 35.06. The earliest detection of this source was at $1.4 \mathrm{GHz}$ with the Cambridge one-mile telescope (Riley 1975). Bondi et al. (1993) studied this source with the Very Large Array (VLA) at $1.4 \& 5 \mathrm{GHz}$ and found a structure with two lobes. The Very Long Baseline Array $(V L B A)$ at $5 \mathrm{GHz}$ found that the large-scale radio galaxy is most likely to be identified as the inner galaxy G3 (Liuzzo et al. 2010). Later, Shulevski et al. (2015) studied this source with the LowFrequency Array $(L O F A R)$ at $62 \mathrm{MHz}$ and found the radio jets have a helical morphology. Biju et al. (2017) has also taken multifrequency radio observations of this source with the Giant Meter-wave Radio Telescope $(G M R T)$ at 610,235 , and $150 \mathrm{MHz}$. They revealed that the large-scale radio structures show 400-kpc scale helically twisted radio jets and outer diffuse lobes.

The radio observations present an interesting helically twisted radio jet structure that could be triggered by the merger of galaxies.
To reveal more details of the merging progress and identify the progenitor galaxy of the large-scale jet structure firmly, higher spatial resolution radio or X-ray data are still needed. X-ray observations can also help us to study the interaction signatures between the hot gas and the radio AGN.

\section{OBSERVATION AND DATA REDUCTION}

The X-ray observation used in this paper was performed with the Chandra Advanced CCD Imaging Spectrometer (ACIS). The observation ID is 18267 (PI: Dharam V. Lal). The exposure time is $43.5 \mathrm{ks}$. We use Chandra Interactive Analysis of Observation (CIAO, version 4.11) and calibration database (CALDB, version 4.7.7) to reprocess the data following a procedure similar to that described in Sun et al. (2009) and Ge et al. (2019). We reprocess a new level 2 event file using the CHANDRA_REPRO script with the VFAINT mode correction. We investigate the light curve in the band $0.5 \sim 9.5 \mathrm{keV}$ from source-free regions to remove the intervals affected by flares, using the DEFLARE script. Such intervals are defined as those deviating more than $3 \sigma$ from the mean rate. We use the WAVDETECT script to detect discrete sources with the point spread function (PSF) map created by the MKPSFMAP script. Blank-sky background files are processed with the BLANKSKY script.

To obtain the background-subtracted and exposure corrected cluster X-ray image, we scale the blank-sky background for each CCD according to the exposure time and count rate in the $9.5-12 \mathrm{keV}$ band. The corresponding exposure maps are generated with the FLUXIMAGE script. The final X-ray image is shown in Fig. 1.

As explained in the Appendix of Sun et al. (2009), there are two basic components in the quiescent Chandra background, the instrumental background, and the cosmic X-ray background (CXB), which are not separately recorded in the blank-sky background file. The CXB would be unphysically scaled if we scale the blank-sky spectrum according to the flux of the instrumental background. After subtracting the scaled blank-sky background, there would still be some residual X-ray sky background in the spectrum.

To get a clean spectrum of the cluster, we use the method of 'double subtraction' to remove the residual sky background. Based on the surface brightness profile (SBP) in Fig. 2, we first choose an off-source region in ACIS-I3 where the cluster emission is negligible and the sky background dominates. Then the spectra are extracted with SPECEXTRACT script for both the off-source region and the cluster region. The blank-sky background is rescaled according to the count rate in the 9.5-12 keV band. Finally, the spectra of the off-source region and the cluster region are jointly fitted with the TBABS* (APEC+PL) +APEC model, with the normalizations connected by the ratio of their areas. The APEC model with absorption represents the cluster emission component. The power-law model with absorption represents the cosmic hard X-ray background composed of unresolved X-ray point sources (Sun et al. 2009). The second APEC model without absorption represents the residual sky background (Vikhlinin et al. 2005). The spectra are fitted jointly with the XSPEC package. We use the AtomDB (version 3.0.8) database of atomic data and the solar abundance tables from Anders \& Grevesse (1989). For the hydrogen column density $N_{\mathrm{H}}$, we adopt the value $1.72 \times 10^{21} \mathrm{~cm}^{-2}$ from the NHtot tool (Willingale et al. 2013). 


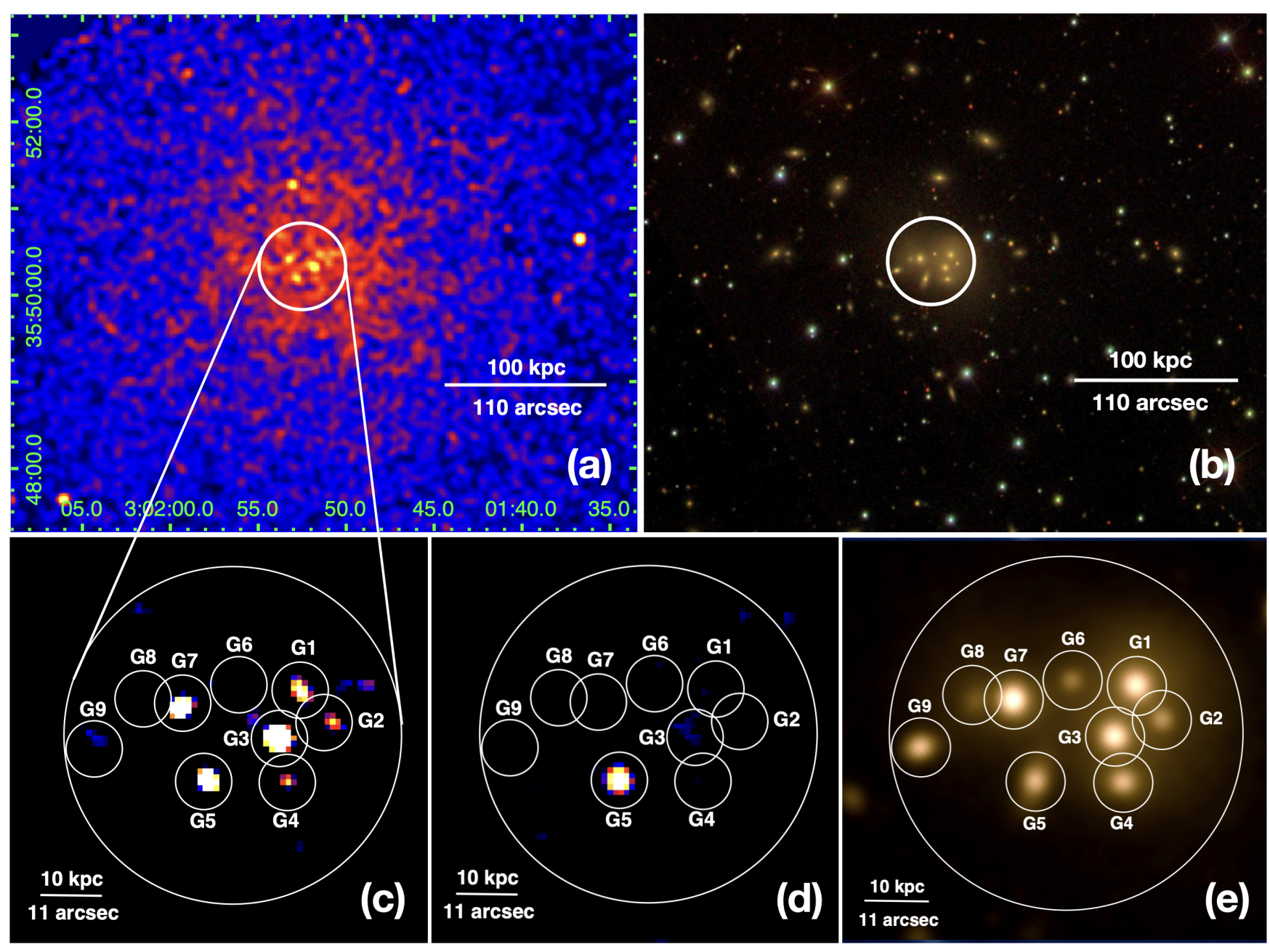

Figure 1. X-ray and optical images of Abell 407 and its central region. The central region hosts nine closely packed galaxies which are embedded within a diffuse stellar halo. This system is possibly a rare cD galaxy precursor assembling in a galaxy cluster environment. ( $a$ ): Chandra background-subtracted and exposure corrected image of A407 in the $0.5-2 \mathrm{keV}$ band. (b): SDSS three-color composite image (blue: $g$-band; green: $r$-band; red: $i$-band) of the same field as the upper left panel. $(c)$ : Soft X-ray $(0.5-2 \mathrm{keV})$ band image of the central galaxies within a circle of $30^{\prime \prime}$ radius. $(d)$ : Hard X-ray (2-7 keV) band image of the central galaxies. (e): SDSS three-color composite image of the central galaxies. All the X-ray images are binned with a size of two $0.492^{\prime \prime}$ pixels and smoothed with a uniform Gaussian kernel of $1.97^{\prime \prime}$.

\section{ANALYSIS AND RESULTS}

\subsection{X-ray properties of central galaxies}

Fig. 1(a) is the background-subtracted and exposure corrected image of A407 in the 0.5-2.0 keV band. The central galaxies in a region with a radius of $30^{\prime \prime}$ are shown in Fig. 1(c,d). We use the circles with a $5^{\prime \prime}$ radius to mark the location of each galaxy. Fig. 1(b) is the corresponding SDSS image. There are seven out of nine optical bright galaxies detected in the soft X-ray $(0.5-2.0 \mathrm{keV})$ image. Among the seven galaxies, the soft X-ray emission from G1, G2, G4, G7, and G9 is weak, while G3 and G5 host the brightest soft X-ray sources in the group. In the hard X-ray (2.0-7.0 keV) image in Fig. 1(d), G5 is the only X-ray bright galaxy.

We derive net X-ray counts for the galaxies in both soft and hard Xray bands. For the count measurement, we use the local background to exclude the contribution from the surrounding hot intracluster medium (ICM). Uncertainties for net counts are given as $1 \sigma$ and upper limits are given as $3 \sigma$. The results are listed in Table 1 . As shown in Table 1, only G6 and G8 are not detected. We give the upper limits of their net counts and exclude them in the following $\mathrm{HR}$ and spectral analysis.

From the counts of the central galaxies, we calculate the hardness ratio for each galaxy. The hardness ratio is defined as $H R=(H-$ $\mathrm{S}) /(\mathrm{H}+\mathrm{S})$, where $\mathrm{S}$ and $\mathrm{H}$ are the net counts in the soft and hard X-ray bands (Wang et al. 2004). The results are shown in Table 1. In the hard X-ray band, since the counts of G2, G7 and G9 are close to the background, we could only give the upper limits of their HR. G5 is the only source that has more counts in the hard X-ray band than the soft band, which may suggest intrinsic absorption for its AGN. The X-ray emission of other galaxies is soft.

We combine the spectra of G1, G2, G3, G4, G7, and G9 as they have similar hardness ratios. We fit the combined spectra with an absorbed APEC thermal model. The redshift and the abundance we adopt are 0.0462 and 1 solar value (Schneider \& Gunn 1982). The fitting result shows that the mean temperature of the X-ray emission from these six galaxies is $1.6_{-1.3}^{+1.2} \mathrm{keV}$. To examine the potential contribution from AGNs and LMXBs, we also add a power-law 
Table 1. Galaxy properties in the central region of Abell 407

\begin{tabular}{|c|c|c|c|c|c|c|c|c|c|c|}
\hline$\overline{\overline{\text { Galaxy }^{a}}}$ & $\begin{array}{l}\text { RA, DEC } \\
(\mathrm{J} 2000)\end{array}$ & $\begin{array}{c}\mathrm{cz}^{b} \\
\left(\mathrm{~km} \mathrm{~s}^{-1}\right)\end{array}$ & $\begin{array}{c}L_{K s^{c}} \\
\left(10^{10} L_{\odot}\right)\end{array}$ & $\begin{array}{c}\text { Mass }^{d} \\
\left(10^{10} M_{\odot}\right)\end{array}$ & $\begin{array}{c}\text { SMBH Mass }^{e} \\
\left(10^{8} M_{\odot}\right)\end{array}$ & $\begin{array}{l}\text { Net counts } \\
(0.5-7 \mathrm{keV})\end{array}$ & $\begin{array}{c}\log L \\
(0.5-2 \mathrm{keV})\end{array}$ & $\begin{array}{c}\log L \\
(2-7 \mathrm{keV})\end{array}$ & $\mathrm{HR}^{f}$ & $\begin{array}{c}\log L_{X} g \\
(0.5-2 \mathrm{keV})\end{array}$ \\
\hline$\overline{\mathrm{G} 1}$ & $03 \mathrm{~h} 01 \mathrm{~m} 51.5 \mathrm{~s}+35 \mathrm{~d} 50 \mathrm{~m} 28.2 \mathrm{~s}$ & $14339(481)$ & 8.5 & 12.63 & $3.88 \pm 1.23$ & $23 \pm 8$ & $40.02_{-0.33}^{+0.19}$ & $39.60_{-0.33}^{+0.19}$ & $-0.61_{-0.31}^{+0.31}$ & 40.82 \\
\hline G2 & $03 \mathrm{~h} 01 \mathrm{~m} 51.2 \mathrm{~s}+35 \mathrm{~d} 50 \mathrm{~m} 22.0 \mathrm{~s}$ & $13600(258)$ & 2.7 & 3.07 & $0.53 \pm 0.44$ & $14 \pm 7$ & $40.25_{-0.24}^{+0.15}$ & $39.83_{-0.24}^{+0.15}$ & $<-0.16$ & 39.07 \\
\hline G4 & $03 \mathrm{~h} 01 \mathrm{~m} 51.7 \mathrm{~s}+35 \mathrm{~d} 50 \mathrm{~m} 12.0 \mathrm{~s}$ & $15030(1172)$ & 4.7 & 6.32 & $0.40 \pm 0.45$ & $13 \pm 7$ & $39.29_{-0}^{+0}$ & $38.87_{-0}^{+0}$ & $-0.39_{-0.52}^{+0.52}$ & 39.93 \\
\hline G5 & $03 \mathrm{~h} 01 \mathrm{~m} 52.9 \mathrm{~s}+35 \mathrm{~d} 50 \mathrm{~m} 12.0 \mathrm{~s}$ & $13199(658)$ & 2.2 & 2.95 & $4.52 \pm 0.74$ & $42 \pm 9$ & $40.38_{-0.12}^{+0.09}$ & $40.51_{-0.11}^{+0.09}$ & $0.21_{-0.21}^{+0.211^{2}}$ & 38.79 \\
\hline G6 & $03 \mathrm{~h} 01 \mathrm{~m} 52.4 \mathrm{~s}+35 \mathrm{~d} 50 \mathrm{~m} 28.9 \mathrm{~s}$ & $13299(558)$ & 0.6 & 0.57 & $0.52 \pm 0.65$ & $<19$ & $<40.68$ & $<41.06$ & - & 36.73 \\
\hline G9 & $03 \mathrm{~h} 01 \mathrm{~m} 54.5 \mathrm{~s}+35 \mathrm{~d} 50 \mathrm{~m} 17.7 \mathrm{~s}$ & $3520(338)$ & 1.9 & 2.22 & $1.34 \pm 0.68$ & $12 \pm 7$ & $39.99_{-1.61}^{+0.30}$ & $39.43_{-1.61}^{+0.30}$ & $<-0.05$ & 38.56 \\
\hline
\end{tabular}

${ }^{a}$ Galaxy names are the same as Biju et al. (2017).

${ }^{b}$ Galaxy velocity values are from NED. The values in parentheses are the absolute value of difference between the galaxy velocities and the HI absorption velocity (13858 $\mathrm{km} \mathrm{s}^{-1}$ ) detected by Shulevski et al. (2015). The HI gas has a similar velocity to that of the host galaxy for 4C 35.06.

${ }^{c}$ The $K_{s}$ band luminosity is derived from the 2MASS point source catalog (PSC). We also measure the total $K_{S}$ band luminosity within $35^{\prime \prime}$ radius of the cluster center, which contains all nine galaxies and their extended emission. The total luminosity is $8.52 \times 10^{11} L_{\odot}$.

${ }^{d}$ The galaxy stellar masses are derived from $L_{K s}$ with the stellar mass-to-light ratio $\log _{10}(M / L)=a_{\lambda}+b_{\lambda} \times$ color (Bell et al. 2003).

$e$ The SMBH masses are calculated by Biju et al. (2017) from bulge stellar velocity dispersion.

${ }^{f} \mathrm{HR}=(\mathrm{H}-\mathrm{S}) /(\mathrm{H}+\mathrm{S}), \mathrm{HR}$ : Hardness ratio; $\mathrm{H}$ : count rate at the $2-7 \mathrm{keV}$ band; $\mathrm{S}$ : count rate at the $0.5-2 \mathrm{keV}$ band.

$g$ The predicted $\log \left(L_{0.5-2 \mathrm{keV}} / \mathrm{erg} \mathrm{s}^{-1}\right)$ from the $L_{K s}-L_{X}$ relation (Sun et al. 2007).

Table 2. The $\beta$-model fitting results of A407's surface brightness profile

\begin{tabular}{l|c}
\hline \hline Parameters & Value \\
\hline$I_{0}\left(10^{-8}\right.$ photons s & -1 \\
$r_{c}(\operatorname{arcsec})$ & $1.75 \pm 0.07$ \\
$\beta$ & $66.7 \pm 2.9$ \\
$\beta$ & $0.42 \pm 0.01$ \\
\hline
\end{tabular}

component with a photon index of 1.7 to fit the combined spectra, as did in Sun et al. (2007). We find the $0.5-2 \mathrm{keV}$ luminosity of the power-law component is at most $30 \%$ of the total $0.5-2 \mathrm{keV}$ luminosity, which suggests that most of the flux arises from thermal coronae.

Due to the limited counts of each galaxy, we estimate their luminosities by fitting a model with fixed parameters. The luminosities are given both in the soft and hard X-ray bands. For G1, G2, G3, G4, G7, and G9, we use the single absorbed APEC model with a temperature of $1.6 \mathrm{keV}$ and 1 solar abundance. For G5, we adopt the single absorbed power-law model with the typical AGN photon index of 1.7. For G6 and G8, we give the estimated upper limits. All results are listed in Table 1.

\subsection{X-ray properties of cluster hot gas}

We extract a SBP from the ICM in Fig. 2. SHERPA is used to fit the SBP with the $\beta$-model (Cavaliere \& Fusco-Femiano 1976):

$I=I_{0}\left(1+x^{2}\right)^{1 / 2-3 \beta}$,

where $x=R / r_{\mathrm{c}}$, and $I_{0}, r_{c}$, and $\beta$ are free parameters. The ICM distribution can be reasonably fit by the $\beta$-model, which is shown in Fig. 2. The best-fitting parameters are in Table 2. We also check the SBP in the sector regions shown in Fig. 4 and the SBPs are similar in different directions.

A global spectrum, extracted excluding the central region, gives a mean X-ray temperature of $2.7 \mathrm{keV}$. This implies a cluster total mass of $M_{500}=1.4 \times 10^{14} M_{\odot}$ and a radius of $r_{500}=774 \mathrm{kpc}$ according to the $M_{500}-T_{500}$ relation from Sun et al. (2009). Here $r_{500}$ refers to the radii within which the cluster mass density is 500 times the universe critical density and $M_{500}$ is the mass within $r_{500}$. We also extract the spectrum from the annuli centered on the X-ray peak to produce

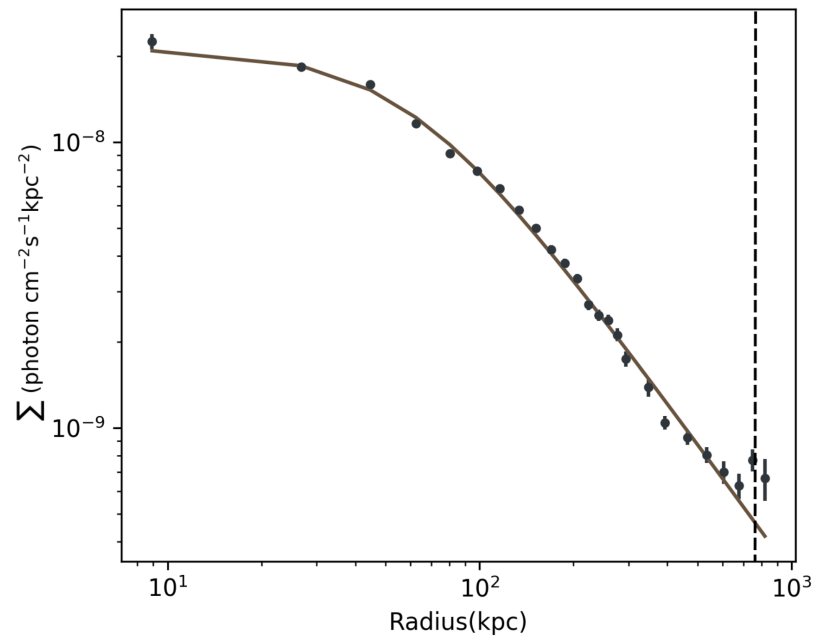

Figure 2. Surface brightness profile of A407 in $0.5-2 \mathrm{keV}$ band. The dashed line marks the $r_{500}$ of A 407 , which is $774 \mathrm{kpc}$. The brightness profile is fitted with the $\beta$-model. The best fitting parameters are listed in Table 2.

the temperature profile as shown in Fig. 3. The region of the central galaxies is excluded to avoid their contamination. The radii of the annuli gradually increase from $30^{\prime \prime}$ to $675^{\prime \prime}$. The radius is increased by steps of $10^{\prime \prime}$ from $30^{\prime \prime}$ to $110^{\prime \prime}$ and $15^{\prime \prime}$ from $110^{\prime \prime}$ to $185^{\prime \prime}$. For the outer 4 large regions, their radii are increased by $30^{\prime \prime}, 60^{\prime \prime}, 100^{\prime \prime}$, and $300^{\prime \prime}$ respectively. As detailed in Section 3, we use the double subtraction method to fit the cluster's spectrum. The energy range is chosen as $0.5-8.0 \mathrm{keV}$. The best-fitting results are shown in Fig. 3. The temperature profile indicates that $\mathrm{A} 407$ has a cool core (CC) and the gas temperature reaches a maximum, $4.18_{-0.50}^{+0.58} \mathrm{keV}$, at $85 \mathrm{kpc}$. We also notice that the temperature rises at $\sim 250 \mathrm{kpc}$, which implies there may exist hot regions.

Alternatively, the spectra from sector regions are fitted to analyze the interaction of radio jets and cluster hot gas. The inner radius of the sector regions is $30^{\prime \prime}$ and the outer radius is $115^{\prime \prime}$. The fitting results are shown in Fig. 4. The regions that contain the radio jets (region 1 and 4 ) have the maximum temperatures which are $4.00_{-0.36}^{+0.36}$ 

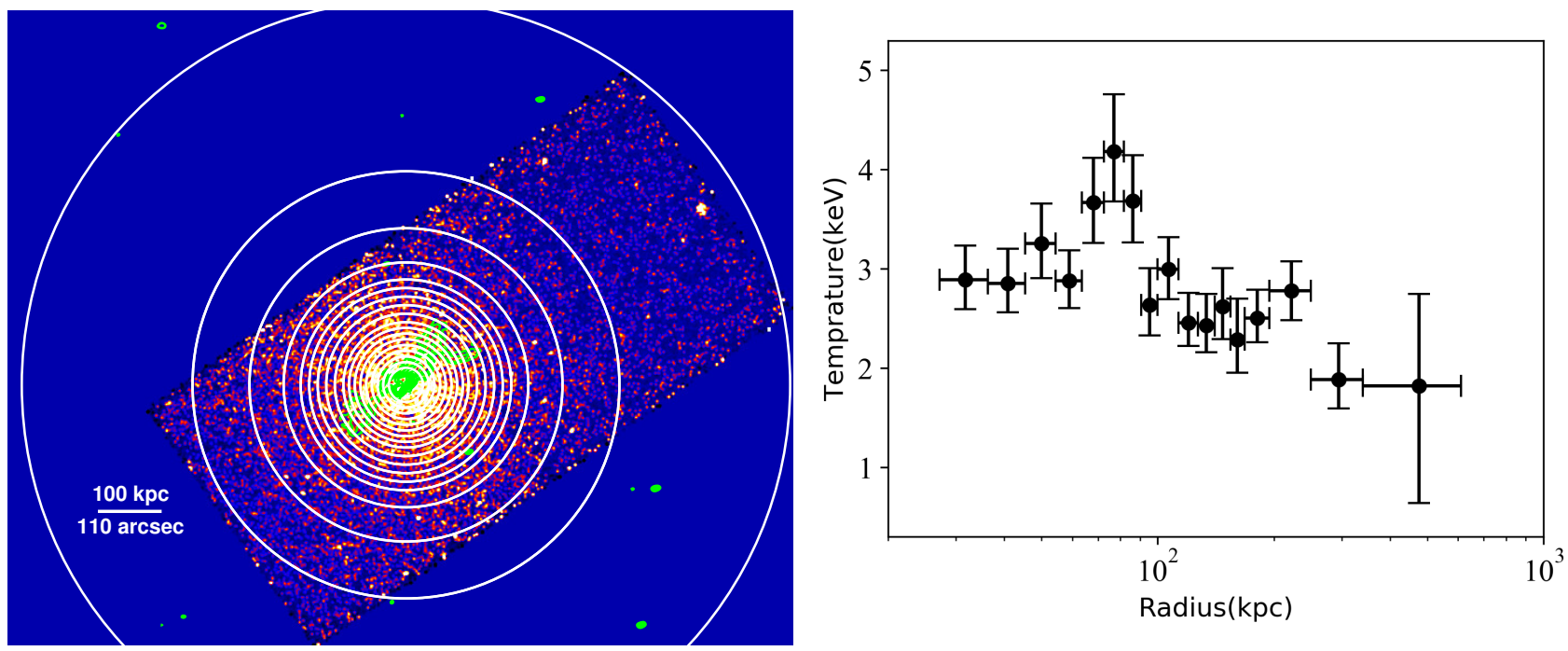

Figure 3. Fitting results of radial annular regions. Left-hand panel: Chandra $0.5-2 \mathrm{keV}$ image overlaid with GMRT $610 \mathrm{MHz}$ contours. The GMRT observation code is 21_066 (PI: Joydeep Bagchi) with 5" FWHM resolution. The X-ray image is binned with a size of two 0.492" pixels and smoothed with a uniform Gaussian kernel of $1.97^{\prime \prime}$. The radius of the rings gradually increases from $30^{\prime \prime}$ to $675^{\prime \prime}$ i.e. 27.2 to 612.2 kpc. Right-hand panel: the temperature as a function of radius shows that $\mathrm{A} 407$ has a weak cool core and the gas temperature reaches a maximum, $4.18_{-0.50}^{+0.58} \mathrm{keV}$, at $85 \mathrm{kpc}$. The temperature and abundance values are given in Table 3.
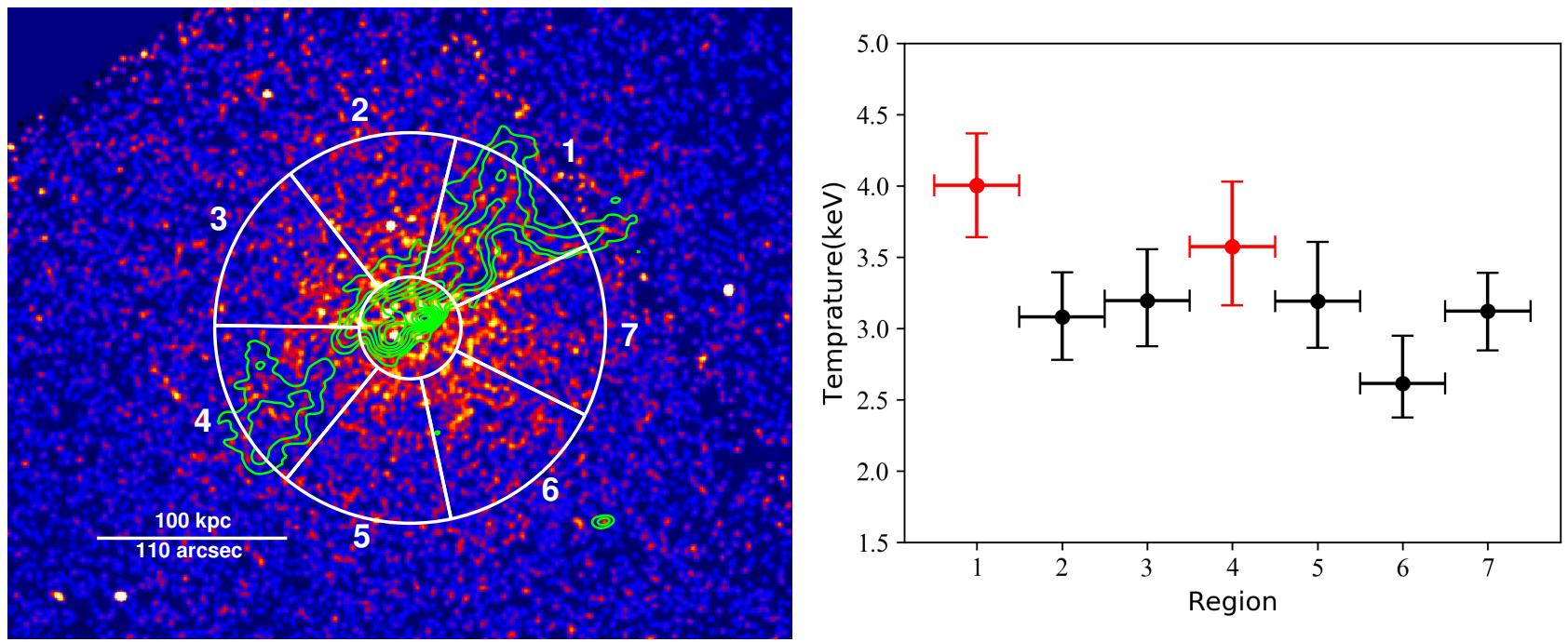

Figure 4. Fitting results of seven panda regions. Left-hand panel: Chandra $0.5-2 \mathrm{keV}$ image overlaid with GMRT $610 \mathrm{MHz}$ contours. The X-ray image is binned with a size of two $0.492^{\prime \prime}$ pixels and smoothed with a uniform Gaussian kernel of $1.97 "$. The inner radius of the sector regions is $30^{\prime \prime}$ and the outer radius is $115^{\prime \prime}$. Right-hand panel: the temperature is plotted against azimuthal region number. The regions that contain the radio jets (plotted in red) have the maximum temperatures which are $4.00_{-0.36}^{+0.36}$ and $3.57_{-0.41}^{+0.45} \mathrm{keV}$ respectively. The temperature and abundance values are listed in Table 4 .

and $3.57_{-0.41}^{+0.45} \mathrm{keV}$ respectively. Region 1 and 7 have the highest abundances, $0.99_{-0.30}^{+0.39}$, and $0.68_{-0.21}^{+0.28}$, which indicates that the higher abundance value may be produced as the jets uplift high abundance gas from the galaxy core.

To analyze the gas structure of the cluster, we derive the ICM temperature map. We extract spectra from ACIS-S3 where the cluster mainly fits. We generate adaptive spatial bins to fit the cluster temperature using 'Contour binning' (Sanders 2006), an algorithm for binning X-ray data by following the surface brightness of the X-ray image. After masking the point sources of the exposure corrected $\mathrm{X}$-ray image, we set the signal-to-noise ratio to 30 , which requires $\sim 900$ background-subtracted counts per bin. We set the geometric constraint value to 1.3, ensuring the shape of bins is not too elongated. As shown in Fig. 5, we use these regions to extract the spectra and response files and fit the gas temperature. We use two regions (region 14 and 15) to cover the radio jet regions and add the fitting result in the temperature map. The detailed temperature of each region are listed in Table 5. We note that regions 14 and 15, where the radio jets lie, have relatively higher temperatures than others.

Entropy is also a useful quantity to study the thermal gas history of a cluster as it reflects ICM global properties. It is defined as $K=T_{X} n_{e}^{-2 / 3}$ (Ponman et al. 1999; Lloyd-Davies et al. 2000), where $T_{X}$ is X-ray temperature and $n_{e}$ is electron density. With the SBP and temperature profile, we can calculate the entropy profile of A407. Assuming spherical symmetry, the cluster's deprojected hydrogen 


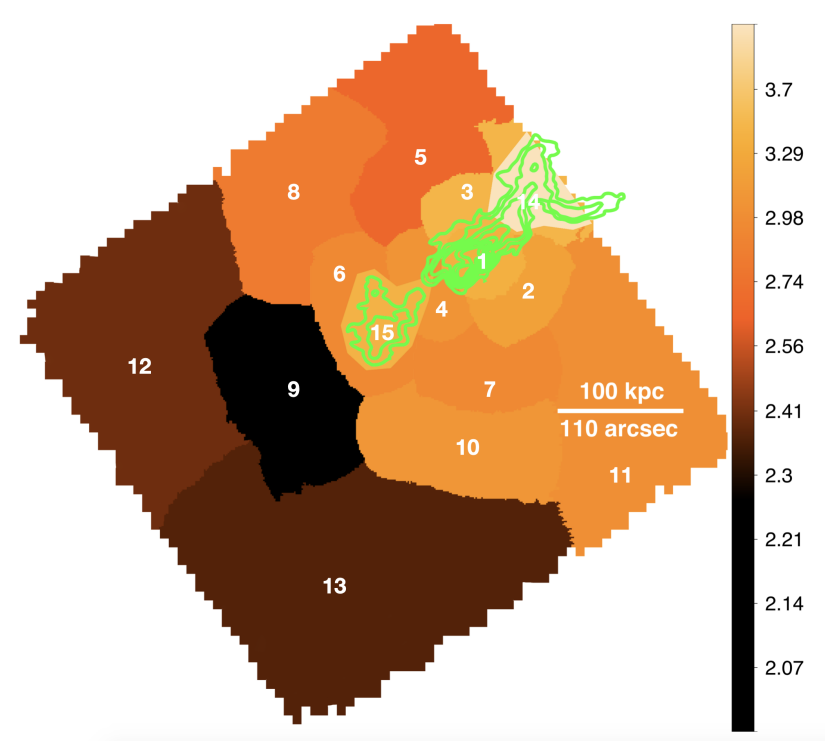

Figure 5. The temperature map generated by Contour Binning and overlaid with GMRT $610 \mathrm{MHz}$ contours. The temperature values are listed in Table 5. The white numbers marked on the picture are the region identifiers. Regions 14 and 15 , where the radio jets lie, have relatively higher temperature than others.

density profile as a function of the physical off-center radius is (e.g. Cavaliere \& Fusco-Femiano 1976; Sarazin 1988):

$n_{\mathrm{H}}=n_{0}\left(1+\frac{r^{2}}{r_{\mathrm{c}}^{2}}\right)^{-\frac{3}{2} \beta}$

where $n_{0}$ represents the central density and we use the equation (4) of Ge et al. (2018) to derive it:

$n_{0}=\frac{3600 \times 180}{\pi} \sqrt{\frac{10^{14} 4 \sqrt{\pi} I_{0} \Gamma(3 \beta)}{\left(\frac{n_{e}}{n_{H}}\right) \frac{f_{x}}{\eta} r_{c} \Gamma(3 \beta-1 / 2)}}$

This equation uses parameters $\left(I_{0}, \beta, r_{c}\right)$ listed in Table 2 from the $\beta$-model fit of the cluster image. $n_{e}$ and $n_{H}$ are the electron and hydrogen number densities and $n_{e} \simeq 1.2 n_{H}$. $\Gamma$ is the gamma function. $\frac{f_{x}}{\eta}$ is the ratio between the X-ray photon flux and the normalization of the APEC model. With the density profile and the temperature map, A407's entropy profile is generated and shown in the middle panel of Fig. 6. We also calculate the central entropy of A407, which is 108 $\mathrm{keVcm}^{2}$.

As detailed in Hudson et al. (2010), from the density profile and temperature profile, we can estimate the average cooling time profile of the gas using:

$t_{\text {cool }}=\frac{3}{2} \frac{\left(n_{\mathrm{e}}+n_{\mathrm{i}}\right) k T}{n_{\mathrm{e}} n_{\mathrm{H}} \Lambda(T, Z)}$

where $n_{\mathrm{i}}$ and $n_{\mathrm{e}}$ are the number density of ions and electrons respectively. $\Lambda(T, Z)$ is determined from the cooling curve in Schure et al. (2009). The cooling time profile is shown in the bottom panel in Fig. 6. From the cooling time profile, we note that the cooling time of the central region is $4.4 \mathrm{Gyr}$.

Hudson et al. (2010) test several parameters to distinguish cool core (CC), weak CC and non-cool-core (NCC) clusters. They find that the central cooling time (measured at $0.048 r_{500}$ ) is the best

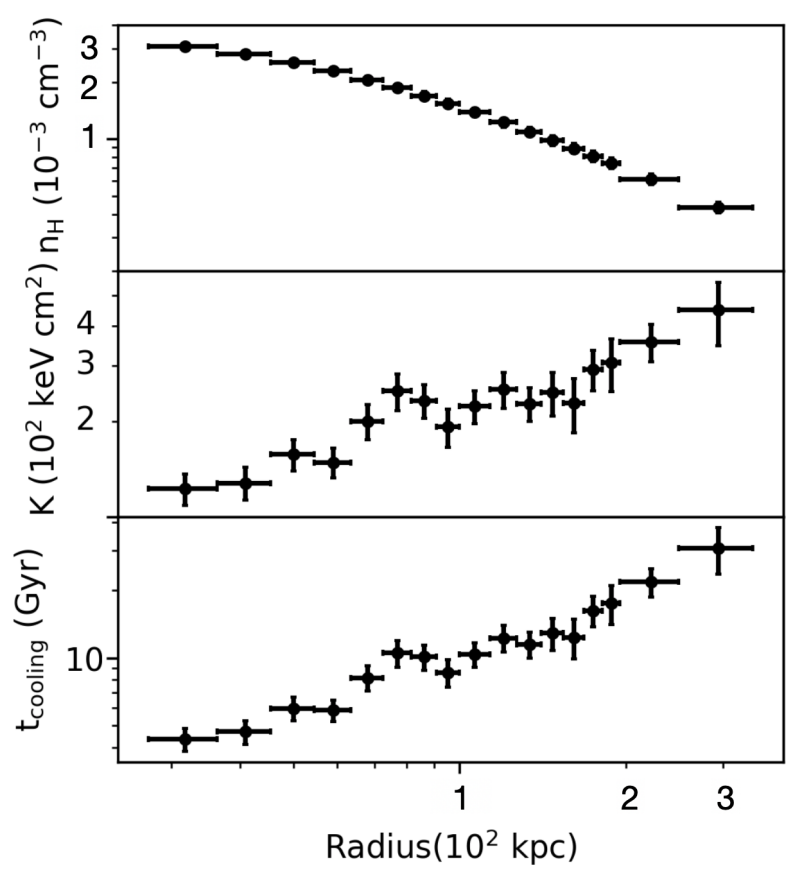

Figure 6. Top panel: Hydrogen density profile of A407. Middle panel: Entropy profile of A407. Bottom panel: cooling time profile of A407. The central region has an entropy of $108 \mathrm{keV} \mathrm{cm}{ }^{2}$ and a cooling time of 4.4 Gyr. See Section 4.2 for details.

parameter to distinguish among the classes for low redshift clusters. Weak CC are characterized as having central cooling times between 1 and 7.7 Gyr. They also suggest the entropy of weak CC clusters is between $\sim 22$ and $\sim 150 \mathrm{keVcm}^{2}$.

As the central cooling time of A407 is 4.4 Gyr and the central entropy is $108 \mathrm{keVcm}^{2}, \mathrm{~A} 407$ is a typical weak CC cluster. The weak $\mathrm{CC}$ size of $\mathrm{A} 407 \mathrm{is} \sim 60 \mathrm{kpc}$ where the cooling time equals the time from $z=1$ to $z=0$.

\section{DISCUSSION}

\subsection{Merging activity of central galaxies}

Two broad classes of physical processes are responsible for the interaction of galaxies in high-density environments. The first class includes the gravitational processes which include all sorts of tidal interactions. The second class includes the hydrodynamic interactions between the galaxy ISM and the hot ICM, such as viscous stripping, ram pressure stripping, and thermal evaporation (Boselli \& Gavazzi 2006).

Previous observations of A407 indicate that it possibly provides unique evidence for the formation of a BCG by mergers. Schneider \& Gunn (1982) suggest this system is a forming $\mathrm{cD}$ by merging events as it harbors a remarkably compact group of galaxies and has an extended stellar envelope. Biju et al. (2017) present the GMRT low-frequency radio images at 610,235 , and $150 \mathrm{MHz}$ and they find a very peculiar twisted jet system which may be caused by the galaxy merging activities. Shulevski et al. (2015) reveal the two episodes of AGN activity with the LOFAR data. The jet precession implies that the AGN is intermittently active as it moves in the dense cluster core. Edwards et al. (2020) investigate the integral spectroscopy of 23 BCGs and determine the age, metallicity, velocity, 
and velocity dispersion of stars in each. Their results are consistent with the scenario that BCG cores formed long ago (with an average age of $13.3 \pm 2.8 \mathrm{Gyr}$ ) and the outer intracluster light (ICL) formed more recently. However, they also notice some local BCGs are still assembling and A407 is one such example.

Chandra's high spatial resolution data reveal more information about the merging process of A407. If it is a rapid merging, we would see signs of gas stripping or no X-ray coronae at all. On the other hand, if the galaxies are in a process of a slow 'merger of equals', we might expect to see X-ray coronae associated with several of the bright galaxies, following the $L_{K s}-L_{X}$ relation (Sun et al. 2007). As detailed in Section 4, seven out of nine optical bright galaxies are detected in the soft X-ray band. However, the detection limit of Chandra with an exposure time of $43 \mathrm{ks}$ is $\sim 10^{40} \mathrm{erg} \mathrm{s}^{-1}$. The $L_{X}$ of galaxies are close to this limit. Therefore, the two remaining galaxies, G6 and G8, may also have faint X-ray corona which could be confirmed with longer exposures.

One possible explanation for the existence of the galaxy corona is that the galaxies are in the process of a 'slow merge' which doesn't strip all the galaxy gas halos. Another explanation is that the galaxies with coronae are far from each other along the line of sight. This leads to the survival of the coronae while some other galaxies, such as G6 and G8, likely lost their halos in the merging progress. However, considering that the velocities of the galaxies (Table 1) vary little, the 'slow merge' model is preferred in A407. We also calculate the $L_{X}$ of each galaxy based on the $L_{K s}-L_{X}$ relation. However, the merging activities may produce the diffuse stellar halos of each galaxy. Therefore, we scale the $L_{K s}$ of each galaxy with a value of 2.35, which is the ratio of the total $K s$ band luminosity inside the radius of $35^{\prime \prime}$ and the sum of $L_{K s}$ of the central nine galaxies. The predicted $L_{X}$ values from $L_{K s}$ are shown in the last column of Table 1 , which are close to the observed $L_{0.5-2 \mathrm{keV}}$ listed in Table 1. Despite the large scatter in the $L_{K} s-L_{X}$ relation, this result supports the 'slow merge' model that coronae of the galaxies are not disrupted entirely in the merging progress.

We calculate the required mass to bind the central galaxies gravitationally with the equation:

$$
M=\frac{3 R \sigma^{2}}{G}
$$

where $\sigma$ is the line of sight velocity dispersion of central galaxies in the rest-frame and $\sigma=584 \mathrm{~km} \mathrm{~s}^{-1}$. The radius $\mathrm{R}$ is $35^{\prime \prime}(31.7 \mathrm{kpc})$ which contains all nine galaxies and their extended emission. The required binding mass is $M_{\text {bind }}=7.5 \times 10^{12} M_{\odot}$. As a comparison, we remove galaxy $\mathrm{G} 4$, which has a most different velocity from those of other central galaxies. The value of $\sigma$ reduces to $413 \mathrm{~km} \mathrm{~s}^{-1}$ and the binding mass reduces to $M_{\text {bind }}=3.8 \times 10^{12} M_{\odot}$.

We also calculate the total mass within $35^{\prime \prime}$ with the dynamical mass-to-light $(M / L)$ relation, which includes the dark matter. Cappellari et al. (2006) investigated the dynamical $M / L$ ratio of elliptical galaxies. They fitted the correlation between the $I$-band $M / L$ and the $K s$-band luminosity:

$$
\left(M / L_{I}\right)=(1.88 \pm 0.20)\left(\frac{L_{K}}{10^{10} L_{K, \odot}}\right)^{0.32 \pm 0.05}
$$

The I-band luminosity within $35^{\prime \prime}$ of A407 is measured from SDSS data $\left(L_{I}=3.1 \times 10^{11} L_{\odot}\right)$ and the $K s$-band luminosity is from the 2 MASS data $\left(L_{k s}=8.5 \times 10^{11} L_{\odot}\right)$. Thus, the dynamical mass within $35^{\prime \prime}$ is $M_{\mathrm{d}}=2.4 \times 10^{12} M_{\odot}$. This value is close to the result that calculated by Biju et al. (2017). They calculated the dynamical mass within the radius of $30^{\prime \prime}$ as $2.2 \times 10^{12} M_{\odot}$ from the halo radial velocity dispersion. Thus, the central dynamical mass of A407 is close to the binding mass calculated above.

Many early-type galaxies (ETGs) have distinctive tidal tails. These tidal tails may be produced during the merging process (Malin \& Carter 1983; van Dokkum 2005; Bennert et al. 2008; Janowiecki et al. 2010). We might also expect such features in the optical band of A407. However, there are no stellar trails detected in the SDSS $r$ band image in Fig. 1(c). Deeper optical observations will help to reveal more details of the merger by detecting such features.

\subsection{The future BCG}

One interesting question is, when the central galaxies merge, what will be the mass of the central BCG? The stellar mass of a galaxy can be calculated from the stellar $M / L$ ratio relation (Bell et al. 2003). We choose a circle with a radius of $35^{\prime \prime}$, which contains all nine galaxies and their extended emission. From the 2MASS $K_{S}$ band image, the total $K_{S}$ band luminosity within this circle is $(8.5 \pm 0.5) \times 10^{11} L_{\odot}$. Thus, the corresponding stellar mass of the future BCG is $(7.4 \pm 0.4) \times 10^{11} M_{\odot}$ using the stellar $M / L$ ratio relation (Bell et al. 2003).

There is a correlation between BCG stellar mass and the host cluster mass. It can be fit with a power-law of the form $M_{\mathrm{BCG}}=$ $\beta M_{\text {Cluster }}^{\alpha}$ where $M_{\mathrm{BCG}}$ is the BCG stellar mass and $M_{\text {Cluster }}$ is $M_{200}$ which represents the cluster mass within $r_{200}$ (Lidman et al. 2012; Bellstedt et al. 2016). Accordingly, we can check whether A407 and its BCG follow this mass relation. The cluster mass $M_{500}=$ $1.4 \times 10^{14} M_{\odot}$ (from the $M_{500}-T_{500}$ relation; Sun et al. (2009)). We then convert $M_{500}$ to $M_{200}$ by assuming the cluster follows an NFW mass profile (Navarro et al. 1997) which gives $r_{500} / r_{200}=0.669$. The resulting $M_{200}$ of A407 is $1.9 \times 10^{14} M_{\odot}$. We find the masses of A407 and its future BCG are consistent with this relation. Thus the predicted value of the mass of the future $\mathrm{BCG}$ is reasonable.

To estimate the merging time of this system, we use the mean merging time-scale relation proposed by Kitzbichler \& White (2008). They investigate the major merger rates based on the Millennium N-body simulation and calibrate the results from deep galaxy surveys. Their work shows that, for samples with stellar masses $\mathrm{M}_{*}>5 \times 10^{9} \mathrm{M}_{\odot}$ and radial velocity differences $\Delta v<3000 \mathrm{~km} \mathrm{~s}^{-1}$, the characteristic mean merging time-scale of galaxy pairs in the low-redshift region $(z \leq 1)$ could be fitted well by:

$$
\left\langle T_{\text {merge }}\right\rangle=3.2 \mathrm{Gyr} \frac{r_{\mathrm{p}}}{50 \mathrm{kpc}}\left(\frac{M_{*}}{4 \times 10^{10} h^{-1} M_{\odot}}\right)^{-0.3}\left(1+\frac{z}{20}\right)
$$

$\left\langle T_{\text {merge }}\right\rangle, r_{\mathrm{p}}$ and $M_{*}$ represent the mean merging time of galaxy pairs, projected separation (kpc), and stellar mass of the galaxy pairs $\left(M_{\odot}\right)$. The projected distance of A407's central galaxies can be obtained from the optical image (Fig. 1). The masses of galaxies are obtained from their $L_{K s}$ which are derived from the 2MASS point source catalog (PSC) (Table 1). However, galaxies are extended in the 2MASS optical image. Thus, these $L_{K s}$ are underestimated. As detailed in Section 5.1, we apply a scale value of 2.35 to each galaxy to derive $L_{K s}$ and stellar mass. We obtain the total mass of each galaxy pair and calculate the merging time-scale by equation 7 . The merging time-scale range is $0.3 \sim 2.3 \mathrm{Gyr}$ which provides a rough estimate of the central merging time of A407. We note that the estimation is based on the two body merging time-scale, the merging process of this compact group is much more complicated and the final merging time is likely larger. 


\subsection{Host of the Radio AGN 4C 35.06}

Which galaxy is the host of 4C 35.06? The VLBA observations by Liuzzo et al. (2010) show pc-scale radio emission centered at the galaxy G3, which suggests that G3 is radio active now. Shulevski et al. (2015) derived the radio luminosity of 4C 35.06 to be $L_{1.4 \mathrm{GHz}}=2.5 \times 10^{24} \mathrm{~W} \mathrm{~Hz}^{-1}$, which makes it a strong radio AGN. They suggested that the rapid movement of galaxy G3 and its episodic AGN radio activities are the reasons for the observed peculiar radio morphology. They also suggest that G3 switched off its radio emission and then restarted while it was moving to its current position, which leads to the offset inner double-lobed morphology. Shulevski et al. (2015) also detected a broad HI absorption feature (FWHM of $288 \mathrm{~km} \mathrm{~s}^{-1}$ ) on top of the strong radio core of 4C 35.06 at a velocity of $\sim 13858 \mathrm{~km} \mathrm{~s}^{-1}$. Assuming the $\mathrm{HI}$ gas has a similar velocity to that of the host galaxy for $4 \mathrm{C} 35.06$, only G2, G3, and G7 have velocities within $300 \mathrm{~km} \mathrm{~s}^{-1}$ of that of the HI absorption feature, as shown in Table 1. Moreover, strong radio AGN are typically associated with massive galaxies. As listed in Table 1, G1, G3, and G7 have the largest galaxy masses. Thus, G3 would be the most likely host from these constraints, as G7 seems to be too far away from the radio center. However, Biju et al. (2017) suggested that the host of the radio AGN is G6 which lies at the center of the principal jet direction. Though G6 is faint in both optical and infrared light, they suggest it may have been stripped of the majority of its outer halo stars in multiple tidal encounters, while still retaining a dense stellar core at the center.

With the Chandra data, we could reveal more information of the central galaxies. Best et al. (2005) and Hardcastle et al. (2007) suggest that radio AGN activity is associated with the cooling of the hot gas from the halos of elliptical galaxies and clusters. Sun (2009) presented a systematic analysis of 152 groups and clusters, and claimed that every BCG with a strong radio AGN $\left(L_{1.4 \mathrm{GHz}}>2 \times 10^{23} \mathrm{~W} \mathrm{~Hz}^{-1}\right)$ has an $\mathrm{X}$-ray CC either in the large cluster $\mathrm{CC}$ class or the galaxy corona class. Since the radio luminosity of $4 \mathrm{C} 35.06$ is $L_{1.4 \mathrm{GHz}}=2.5 \times 10^{24} \mathrm{~W} \mathrm{~Hz}^{-1}$, which is larger than $2 \times 10^{23} \mathrm{~W} \mathrm{~Hz}^{-1}$, it should have an X-ray CC. As mentioned in Section 4, A407 is a weak CC cluster and G3 has the most prominent $\mathrm{X}$-ray corona and lies closer to the radio core. Therefore, we suggest G3 is the most likely host of the luminous radio AGN. Of course, an ambiguous determination of the host would require radio images at higher angular resolution. As G5 hosts the brightest X-ray AGN in this group and likely hosts a massive SMBH from the analysis by Biju et al. (2017), we cannot exclude the possibility that G5 also contributes to the radio emission of 4C 35.06.

\subsection{Feedback from AGN}

AGN can release tremendous amounts of energy to their surroundings. Boehringer et al. (1993) noted the existence of radio-filled cavities in NGC1275 based on ROSAT observations. McNamara et al. (2000) found a close association between the radio lobes and X-ray cavities in Hydra A which could suggest the gas was displaced as the radio lobes expanded. Thus X-ray cavities can provide a direct way to measure the energy injected by AGN (Churazov et al. 2000, 2001).

The energy from radio jets are near those required to offset radiative cooling (Fabian 2012; McNamara \& Nulsen 2012). Nulsen et al. (2009) studied a sample of nearby giant elliptical galaxies from Chandra observations. Their results show that the average power injected by AGN is sufficient to stop the hot atmospheres from cooling. Not only the temperature, heavy elements can also be transported from

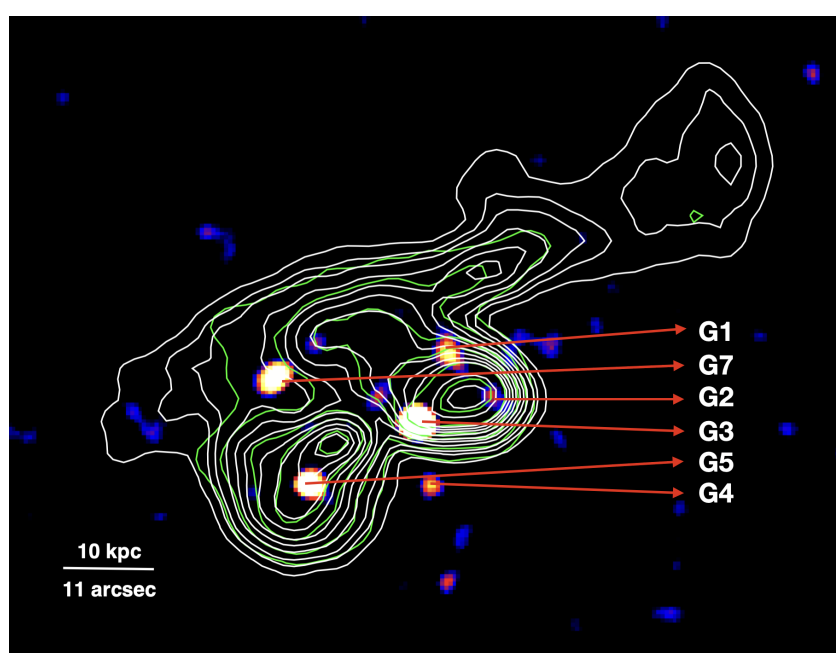

Figure 7. Chandra $0.5-2 \mathrm{keV}$ image overlaid with $V L A 1.5 \mathrm{GHz}$ (white) and $4.9 \mathrm{GHz}$ (green) contours.

the core by the large-scale outflows and change the ICM abundance (McNamara \& Nulsen 2007; Simionescu et al. 2008; Kirkpatrick et al. 2011; Kirkpatrick \& McNamara 2015).

Biju et al. (2017) suggested the possibility that radio jets in this source might be heating and interacting with the ICM via a jet-mode feedback. The temperature map of A407 does indicate the presence of feedback features including heating and abundance enrich. As shown in Fig. 4, the regions that overlap with the radio jets (region 1 and 4) have the maximum temperatures i.e. $4.0_{-0.4}^{+0.4}$ and $3.6_{-0.4}^{+0.5} \mathrm{keV}$. This implies that the gas around the radio jets is heated by the feedback. As can be seen from Table 4, region 1 and 7, which include the northwest radio jet, have the highest abundances, $1.0_{-0.3}^{+0.4}$, and $0.7_{-0.2}^{+0.3}$ respectively. The increase in temperature and abundance along the jet direction is also seen in Fig. 5 and Table 5.

The power of cavities can provide a lower limit to the jet energy as it is a direct evidence for AGN heating (Bîrzan et al. 2008). Bîrzan et al. (2008), Cavagnolo et al. (2010), and O'Sullivan et al. (2011) investigate the scaling relationship between mechanical jet power and radio luminosity. Based on their work, we estimate the jet power's lower limit in A407 from its $1.4 \mathrm{GHz}$ radio luminosity $\left(L_{1.4 \mathrm{GHz}}=2.5 \times 10^{24} \mathrm{~W} \mathrm{~Hz}^{-1}\right)$. The lower limit of the mechanical jet power is $1.0 \times 10^{44} \mathrm{erg} \mathrm{s}^{-1}$.

As detailed in Section 4.2, no cavity structure has been found in A407 yet. It may need deeper X-ray observation to confirm. Assuming there exist two cavities with the same scale of the $610 \mathrm{MHz}$ radio contour in Fig. 4 and their shape could be approximated by spheres with the radius of $40 \mathrm{kpc}$, we can estimate the mechanical $\mathrm{pV}$ energy of the cavities based on the gas temperature and density profile (McNamara \& Nulsen 2012). The total mechanical energy of the two cavities is $5.8 \times 10^{59} \mathrm{erg}$. As the mechanical jet power is $1.0 \times 10^{44} \mathrm{erg} \mathrm{s}^{-1}$, it would take $0.18 \mathrm{Gyr}$ for the radio AGN to supply the cavities' mechanical pV energy. For comparison, the central cooling time of A407 is 4.4 Gyr.

Entropy can also reflect ICM global properties. Cavagnolo et al. (2009) present radial entropy profiles for 239 clusters from the Chandra X-ray Observatory Data Archive. They find that most entropy profiles are well fitted by a power law at large radii and a constant value at small radii: $K(r)=K_{0}+K_{100}(r / 100 \mathrm{kpc})^{\alpha} . K_{0}$ is the typical excess of core entropy above the best-fitting power law found at larger radii. For clusters with temperature $T_{X}<4 \mathrm{keV}$, the $K_{0}$ distribution peaks at $K_{0} \sim 15 \mathrm{keV} \mathrm{cm}^{2}$. For clusters with tempera- 
ture $T_{X}>8 \mathrm{keV}$, the $K_{0}$ distribution peaks at $K_{0} \sim 150 \mathrm{keV} \mathrm{cm}^{2}$. The mean temperature of A407 is $2.7 \mathrm{keV}$ and the entropy profile is shown in Fig. 6. From the figure, we notice that the entropy profile of A407 is relatively higher compared to other clusters with temperatures lower than $T_{X}<4 \mathrm{keV}$ in Cavagnolo et al. (2009). The entropy at small radii is $\sim 200 \mathrm{keV} \mathrm{cm}^{2}$. The higher entropy in A407 at small radii might be caused by the AGN feedback.

In summary, A407 shows evidence that its ICM temperature, abundance, and entropy are affected by AGN feedback, most likely from the radio jets.

\section{SUMMARY}

A407 contains a compact group of galaxies in its central region. We study A407 with the Chandra observation combined with previous radio and optical observations. Our major results and conclusions are:

- A407 has a weak CC within $60 \mathrm{kpc}$. The central region of A407 has a cooling time of $4.4 \mathrm{Gyr}$ and an entropy of $108 \mathrm{keVcm}^{2}$. The $M_{200}$ of A407 is $1.9 \times 10^{14} M_{\odot}$.

- Due to the existence of the central galaxy X-ray coronae, we suggest the central galaxies are in the process of a 'slow merge'. When these galaxies merge, we predict the future BCG's stellar mass would be $7.4 \times 10^{11} M_{\odot}$ and the merging time-scale range is $0.3 \sim 2.3$ Gyr. The predicted BCG mass follows the mass relation between BCGs and host clusters (e.g. Lidman et al. 2012; Bellstedt et al. 2016).

- For galaxies in the central region of A407, G3 has the most prominent X-ray thermal corona. Combining the results of X-ray and radio observations, we suggest galaxy G3 is the host of radio AGN 4C 35.06 .

- We also find evidence of AGN feedback in the hot gas of A407. The regions that overlap with the radio jets have higher temperatures and abundance than other regions. The entropy of A407's center is also relatively higher than other clusters which may be due to the effects of AGN feedback.

In summary, A407 has a very unusual cluster core in optical, which is populated by a cluster of galaxies instead of a single dominant galaxy as in most clusters. We witness it in a crucial formation epoch accompanied by the helically twisted jets and merging features which have the potential of clarifying how BCGs originate. We present a study focused on the X-ray band to reveal merging details of the central galaxies and implications of the AGN feedback activities. A407 suggests the scenario in which AGN activity can be triggered during the formation of the cluster BCG. Deeper X-ray observations would reveal more details of the merging and AGN feedback process. Further optical observation would help to confirm if there exist any stellar tidal trails. More cases, from both low and high redshifts, are needed to reveal more detail about the BCG formation processes.

\section{ACKNOWLEDGEMENTS}

We thank the referee, Dr. Joydeep Bagchi, for comments. The scientific results reported in this article are based on the observation made by the Chandra X-ray Observatory, ObsIDs 18267, which is operated by the Smithsonian Astrophysical Observatory (SAO) for and on behalf of the National Aeronautics Space Administration (NASA). This publication makes use of data products from the Two Micron All Sky Survey, which is a joint project of the University of Massachusetts and the Infrared Processing and Analysis Center/California Institute of Technology, funded by the National Aeronautics and Space Administration and the National Science Foundation. We also present data obtained with the Giant Meter-wave Radio Telescope (GMRT), which is run by the National Centre for Radio Astrophysics of the Tata Institute of Fundamental Research. We use the images from $S D S S$ and the funding for SDSS is provided by Alfred P. Sloan Foundation, the U.S. Department of Energy Office of Science, and the Participating Institutions. We also made use of the NASA/IPAC Extragalactic Database (NED) which is operated by the Jet Propulsion Laboratory, California Institute of Technology, under contract with NASA. D.V.L. acknowledges the support of the Department of Atomic Energy, Government of India, under project no.12-R\&DTFR-5.02-0700. L.J. is supported by NSFC grant U1531248 and BICCAS grant 114332KYSB20180013. W.F. and C.J. acknowledge support from the Smithsonian Institution and the Chandra High Resolution Camera Project through NASA contract NAS8-03060.

\section{DATA AVAILABILITY}

The Chandra raw data used in this paper are available to download at the Chandra Data Archive ${ }^{1}$ and HEASARC Data Archive ${ }^{2}$ website. The reduced data underlying this paper will be shared on reasonable requests to the corresponding authors.

\section{REFERENCES}

Anders E., Grevesse N., 1989, Geochimica Cosmochimica Acta, 53, 197

Beers T. C., Geller M. J., 1983, ApJ, 274, 491

Bell E. F., McIntosh D. H., Katz N., Weinberg M. D., 2003, ApJS, 149, 289

Bell E. F., et al., 2006, ApJ, 640, 241

Bellstedt S., et al., 2016, MNRAS, 460, 2862

Bennert N., Canalizo G., Jungwiert B., Stockton A., Schweizer F., Peng C. Y., Lacy M., 2008, ApJ, 677, 846

Best P. N., Kauffmann G., Heckman T. M., Brinchmann J., Charlot S., Ivezić Ž., White S. D. M., 2005, MNRAS, 362, 25

Biju K. G., et al., 2017, MNRAS, 471, 617

Bîrzan L., McNamara B. R., Nulsen P. E. J., Carilli C. L., Wise M. W., 2008, ApJ, 686, 859

Boehringer H., Voges W., Fabian A. C., Edge A. C., Neumann D. M., 1993, MNRAS, 264, L25

Bondi M., Gregorini L., Padrielli L., Parma P., 1993, A\&AS, 101, 431

Boselli A., Gavazzi G., 2006, PASP, 118, 517

Cappellari M., et al., 2006, MNRAS, 366, 1126

Cavagnolo K. W., Donahue M., Voit G. M., Sun M., 2009, ApJS, 182, 12

Cavagnolo K. W., McNamara B. R., Nulsen P. E. J., Carilli C. L., Jones C., Bîrzan L., 2010, ApJ, 720, 1066

Cavaliere A., Fusco-Femiano R., 1976, A\&A, 500, 95

Churazov E., Forman W., Jones C., Böhringer H., 2000, A\&A, 356, 788

Churazov E., Brüggen M., Kaiser C. R., Böhringer H., Forman W., 2001, ApJ, 554, 261

Cowie L. L., Binney J., 1977, ApJ, 215, 723

De Lucia G., Blaizot J., 2007, MNRAS, 375, 2

Dressler A., 1978, ApJ, 223, 765

Dubinski J., 1998, ApJ, 502, 141

Edwards L. O. V., et al., 2020, MNRAS, 491, 2617

Fabian A. C., 1994, ARA\&A, 32, 277

Fabian A. C., 2012, ARA\&A, 50, 455

Ge C., Wang Q. D., Burchett J. N., Tripp T. M., Sun M., Li Z., Gu Q., Ji L., 2018, MNRAS, 481, 4111

\footnotetext{
1 https://cxc.harvard.edu/cda/

2 https://heasarc.gsfc.nasa.gov/docs/archive.html
} 
Ge C., Sun M., Rozo E., Sehgal N., Vikhlinin A., Forman W., Jones C., Nagai D., 2019, MNRAS, 484, 1946

Hardcastle M. J., Evans D. A., Croston J. H., 2007, MNRAS, 376, 1849

Hausman M. A., Ostriker J. P., 1978, ApJ, 224, 320

Hudson D. S., Mittal R., Reiprich T. H., Nulsen P. E. J., Andernach H., Sarazin C. L., 2010, A\&A, 513, A37

Janowiecki S., Mihos J. C., Harding P., Feldmeier J. J., Rudick C., Morrison H., 2010, ApJ, 715, 972

Jones C., Forman W., 1984, ApJ, 276, 38

Khochfar S., Silk J., 2009, ApJ, 700, L21

Kirkpatrick C. C., McNamara B. R., 2015, MNRAS, 452, 4361

Kirkpatrick C. C., McNamara B. R., Cavagnolo K. W., 2011, ApJ, 731, L23

Kitzbichler M. G., White S. D. M., 2008, MNRAS, 391, 1489

Kravtsov A. V., Borgani S., 2012, ARA\&A, 50, 353

Lidman C., et al., 2012, MNRAS, 427, 550

Liu F. S., Mao S., Deng Z. G., Xia X. Y., Wen Z. L., 2009, MNRAS, 396, 2003

Liuzzo E., Giovannini G., Giroletti M., Taylor G. B., 2010, A\&A, 516, A1

Lloyd-Davies E. J., Ponman T. J., Cannon D. B., 2000, MNRAS, 315, 689

Malin D. F., Carter D., 1983, ApJ, 274, 534

McConnell N. J., Ma C.-P., Gebhardt K., Wright S. A., Murphy J. D., Lauer

T. R., Graham J. R., Richstone D. O., 2011, Nature, 480, 215

McNamara B. R., Nulsen P. E. J., 2007, ARA\&A, 45, 117

McNamara B. R., Nulsen P. E. J., 2012, New Journal of Physics, 14, 055023

McNamara B. R., et al., 2000, ApJ, 534, L135

Merritt D., 1985, ApJ, 289, 18

Navarro J. F., Frenk C. S., White S. D. M., 1997, ApJ, 490, 493

Nulsen P., Jones C., Forman W., Churazov E., McNamara B., David L., Murray S., 2009, in Heinz S., Wilcots E., eds, American Institute of Physics Conference Series Vol. 1201, The Monster's Fiery Breath: Feedback in Galaxies, Groups, and Clusters. pp 198-201 (arXiv:0909.1809), doi: $10.1063 / 1.3293033$

O’Sullivan E., Giacintucci S., David L. P., Gitti M., Vrtilek J. M., Raychaudhury S., Ponman T. J., 2011, ApJ, 735, 11

Ostriker J. P., Tremaine S. D., 1975, ApJ, 202, L113

Ponman T. J., Cannon D. B., Navarro J. F., 1999, Nature, 397, 135

Riley J. M., 1975, MNRAS, 170, 53

Sanders J. S., 2006, MNRAS, 371, 829

Sarazin C. L., 1988, X-ray emission from clusters of galaxies

Schneider D. P., Gunn J. E., 1982, ApJ, 263, 14

Schure K. M., Kosenko D., Kaastra J. S., Keppens R., Vink J., 2009, A\&A, 508,751

Shulevski A., et al., 2015, A\&A, 579, A27

Simionescu A., Werner N., Finoguenov A., Böhringer H., Brüggen M., 2008, A\&A, 482, 97

Sun M., 2009, ApJ, 704, 1586

Sun M., Jones C., Forman W., Vikhlinin A., Donahue M., Voit M., 2007, ApJ, 657, 197

Sun M., Voit G. M., Donahue M., Jones C., Forman W., Vikhlinin A., 2009, ApJ, 693, 1142

Thomas J., Ma C.-P., McConnell N. J., Greene J. E., Blakeslee J. P., Janish R., 2016, Nature, 532, 340

Tovmassian H. M., Andernach H., 2012, MNRAS, 427, 2047

Tremaine S., 1990, The origin of central cluster galaxies.. pp 394-405

Vikhlinin A., Markevitch M., Murray S. S., Jones C., Forman W., Van Speybroeck L., 2005, ApJ, 628, 655

Voit G. M., 2005, Reviews of Modern Physics, 77, 207

Von Der Linden A., Best P. N., Kauffmann G., White S. D. M., 2007, MNRAS, 379,867

Wang J. X., Malhotra S., Rhoads J. E., Norman C. A., 2004, ApJ, 612, L109

West M. J., 1994, MNRAS, 268, 79

Willingale R., Starling R. L. C., Beardmore A. P., Tanvir N. R., O’Brien P. T., 2013, MNRAS, 431, 394

Zwicky F., Zwicky M. A., 1971, Catalogue of selected compact galaxies and of post-eruptive galaxies

van Dokkum P. G., 2005, AJ, 130, 2647 
Table 3. The fitting results of the radial annular regions.

\begin{tabular}{|c|c|c|c|c|c|c|c|c|c|}
\hline Radius (arcsec) & $30-40$ & $40-50$ & $50-60$ & $60-70$ & $70-80$ & $80-90$ & $90-100$ & $100-110$ & $110-125$ \\
\hline Radius (kpc) & $27.2-36.2$ & $36.2-45.3$ & $45.3-54.4$ & $54.4-63.4$ & $63.4-72.5$ & $72.5-81.6$ & $81.6-90.7$ & $90.7-99.7$ & $99.7-113.3$ \\
\hline Temperature $(\mathrm{keV})$ & $2.89_{-0.30}^{+0.34}$ & $2.85_{-0.29}^{+0.35}$ & $3.25_{-0.35}^{+0.40}$ & $2.88_{-0.27}^{+0.31}$ & $3.67_{-0.41}^{+0.45}$ & $4.18_{-0.50}^{+0.58}$ & $3.68_{-0.42}^{+0.46}$ & $2.64_{-0.31}^{+0.37}$ & $2.99_{-0.30}^{+0.33}$ \\
\hline Abundance & $0.32_{-0.16}^{+0.23}$ & $0.31_{-0.15}^{+0.21}$ & $0.18_{-0.14}^{+0.19}$ & $\begin{array}{r}0.47_{-0.19}^{+0.25} \\
\end{array}$ & $\begin{array}{l}0.60_{-0.26}^{+0.35} \\
\end{array}$ & $0.76_{-0.32}^{+0.46}$ & $\begin{array}{l}0.67_{-0.28}^{+0.38} \\
\end{array}$ & $0.17_{-0.13}^{+0.19}$ & $\begin{array}{r}0.50_{-0.19}^{+0.25} \\
\end{array}$ \\
\hline Radius (arcsec) & $125-140$ & $140-155$ & $155-170$ & $170-185$ & $185-215$ & $215-275$ & $275-375$ & $375-675$ & \\
\hline Radius (kpc) & $113.3-126.9$ & $126.9-140.5$ & $140.5-154.1$ & $154.1-167.7$ & $167.7-195.1$ & $195.0-249.4$ & 249.4-340.1 & $340.1-612.2$ & \\
\hline Temperature (keV) & $2.46_{-0.23}^{+0.30}$ & $2.43_{-0.27}^{+0.32}$ & $2.62_{-0.33}^{+0.39}$ & $2.29_{-0.33}^{+0.42}$ & $2.51_{-0.25}^{+0.28}$ & $2.78_{-0.29}^{+0.30}$ & $1.89_{-0.29}^{+0.36}$ & $1.82_{-1.18}^{+0.92}$ & \\
\hline Abundance & $0.18_{-0.11}^{+0.14}$ & $0.54_{-0.23}^{+0.34}$ & $0.18_{-0.13}^{+0.19}$ & $0.13_{-0.11}^{+0.19}$ & $0.26_{-0.12}^{+0.16}$ & $0.53_{-0.17}^{+0.22}$ & $0.24_{-0.18}^{+0.30}$ & $0.23_{-0.23}^{+0.90}$ & \\
\hline
\end{tabular}

Table 4. The fitting results of the 7 panda shape regions.

\begin{tabular}{lccccccc}
\hline \hline region & 1 & 2 & 3 & 4 & 5 & 6 & 7 \\
\hline Temperature $(\mathrm{keV})$ & $4.00_{-0.36}^{+0.36}$ & $3.08_{-0.30}^{+0.31}$ & $3.19_{-0.32}^{+0.36}$ & $3.57_{-0.41}^{+0.45}$ & $3.19_{-0.33}^{+0.42}$ & $2.62_{-0.24}^{+0.33}$ & $3.12_{-0.28}^{+0.27}$ \\
\hline Abundance & $0.99_{-0.30}^{+0.39}$ & $0.20_{-0.13}^{+0.16}$ & $0.31_{-0.16}^{+0.22}$ & $0.31_{-0.18}^{+0.23}$ & $0.30_{-0.16}^{+0.22}$ & $0.17_{-0.10}^{+0.14}$ & $0.68_{-0.21}^{+0.28}$ \\
\hline
\end{tabular}

Table 5. The fitting results of the regions generated by Contour Binning.

\begin{tabular}{|c|c|c|c|c|c|c|c|c|}
\hline region & $\overline{1}$ & $\overline{2}$ & 3 & $\overline{4}$ & $\overline{5}$ & $\overline{76}$ & $\overline{77}$ & $\overline{88}$ \\
\hline Temperature (keV) & $3.36^{+0.37}$ & $3.18^{+0.33}$ & $3.44^{+0.27}$ & $3.04^{+0.31}$ & $2.66^{+0.26}$ & $3.09^{+0.38}$ & $2.94^{+0.36}$ & $2.81^{+0.40}$ \\
\hline Abundance & $0.48_{-0.20}^{+0.30}$ & $0.25_{-0.13}^{+0.30}$ & $0.62_{-0.17}^{+0.26}$ & $0.42_{-0.17}^{+0.22}$ & $0.40_{-0.21}^{+0.21}$ & $0.39_{-0.16}^{+0.36}$ & $0.19_{-0.12}^{+0.31}$ & $0.30_{-0.11}^{+0.37}$ \\
\hline Temperature $(\mathrm{keV})$ & $2.01_{-0.22}^{+0.31}$ & $3.07_{-0.38}^{+0.46}$ & $2.99_{-0.28}^{+0.29}$ & $2.40_{-0.37}^{+0.45}$ & $2.36_{-0.34}^{+0.53}$ & $4.16_{-0.45}^{+0.52}$ & $3.53_{-0.40}^{+0.47}$ & \\
\hline Abundance & $0.41_{-0.14}^{-0.22}$ & $0.10_{-0.07}^{+0.38}$ & $0.40_{-0.20}^{+0.28}$ & $0.21_{-0.14}^{+0.37}$ & $0.27_{-0.15}^{-0.34}$ & $1.55_{-0.56}^{-0.45}$ & $0.38_{-0.20}^{+0.40}$ & \\
\hline
\end{tabular}

